\title{
25 Research Soure \\ Prediction of Best Corrected Visual Acuity with Hard Contact Lenses for Keratoconus Patients
}

\author{
Kazuyoshi Magome \\ Ohshima Eye Hospital \\ Naoyuki Morishige ( $\nabla$ morishig@corneajp.com ) \\ Ohshima Eye Hospital \\ Hirofumi Nagai \\ Ohshima Eye Hospital \\ Akifumi Ueno \\ Ohshima Eye Hospital \\ Takaaki Matsui \\ Ohshima Eye Hospital \\ Eiichi Uchio \\ Fukuoka University School of Medicine
}

\section{Research Article}

Keywords: keratoconus, anterior segment optical coherent tomography, hard contact lenses, visual acuity

Posted Date: August 31st, 2021

DOl: https://doi.org/10.21203/rs.3.rs-849329/v1

License: (c) (i) This work is licensed under a Creative Commons Attribution 4.0 International License.

Read Full License 


\section{Abstract}

Background: To develop a prediction formula for best corrected visual acuity with hard contact lenses (HCL-BCVA) and to identify clinical factors linearly related to HCL-BCVA in keratoconus patients.

Methods: This retrospective study examined clinical data derived from 198 eyes of 131 keratoconus patients. The subjects were divided into a development group (102 eyes of 68 subjects) and a validation group (96 eyes of 63 subjects) on the basis of the date of their examination. HCL-BCVA measurement and anterior segment-optical coherence tomography (AS-OCT) were performed. A prediction formula for HCL-BCVA based on AS-OCT measurements was then developed. Single regression analysis was performed to identify clinical factors linearly related to HCL-BCVA.

Results: Stepwise multiple regression analysis yielded a prediction formula for HCL-BCVA in keratoconus patients, with the correlation coefficient of the multiple regression equation being $0.728\left(R^{2}=0.530\right)$ for the development group. Application of the prediction formula to the validation group yielded a correlation coefficient for the multiple regression equation of $0.641\left(R^{2}=0.411\right)$. Single regression analysis identified anterior corneal refractive power, posterior corneal refractive power, and high-order aberrations as factors that are linearly correlated with HCL-BCVA, with $R$ values of $0.606,-0.617$, and 0.506 , respectively.

Conclusion: HCL-BCVA in keratoconus patients was predictable on the basis of AS-OCT measurements. Cutoff values for clinical factors found to correlate with HCL-BCVA may prove informative with regard to treatment options to maintain a favorable visual acuity in keratoconus patients.

\section{Introduction}

Keratoconus is one of the most common corneal diseases and is characterized by central corneal protrusion and thinning that result in pronounced corneal irregular astigmatism. ${ }^{1-7}$ Visual correction with glasses or soft contact lenses is usually applied to individuals with mild keratoconus. However, for those with severe keratoconus, visual correction with hard contact lenses (HCLs) is necessary to obtain acceptable visual acuity. Rigid gas permeable contact lenses, which can cancel the anterior corneal irregular astigmatism and anterior corneal higher aberration, are thus prescribed for most patients with keratoconus. ${ }^{8-15}$ In the case of such patients who are not able to obtain a comfortable fit with conventional HCLs, modified HCLs such as multicurved or scleral lenses have recently been applied. ${ }^{16-18}$ However, corneal posterior irregular astigmatism also impairs vision in keratoconus patients and cannot be corrected by any type of $\mathrm{HCL}$, which can result in a poor best corrected visual acuity (BCVA) even if HCL correction is applied. $8,9,13,15,19,20$ Some individuals with keratoconus are thus not able to achieve a visual acuity that does not have a negative impact on their quality of life.

Corneal cross-linking (CXL) treatment is administered to prevent the progression of keratoconus. ${ }^{21}$ The indications for $\mathrm{CXL}$ vary among countries. In Japan, the key conditions for $\mathrm{CXL}$ include progression with more than 1.0D of subjective spherical refraction, more than $1.0 \mathrm{D}$ of subjective astigmatism, more than 
1.0D of corneal refractive power detected by corneal topography, or a decrease of less than $0.1 \mathrm{~mm}$ of HCL base curve value. ${ }^{22}$ The purpose of CXL is to halt the progression of keratoconus, and it should be performed while the patient is still able to achieve an adequate visual acuity with HCLs. However, progression of keratoconus to a point at which good visual acuity cannot be achieved even with HCLs may represent a missed opportunity for application of CXL. Prevention of such a missed opportunity may necessitate a different approach to determining the indications for CXL.

We have now developed a formula to predict BCVA with HCLs (HCL-BCVA) for keratoconus patients on the basis of data for corneal parameters measured by anterior segment-optical coherence tomography (AS-OCT). We also analyzed the relation between actual HCL-BCVA and corneal parameters to identify threshold values for loss of an adequate level of HCL-BCVA. Our results suggest that evaluation of keratoconus by AS-OCT can inform treatment strategy including the application of CXL to prevent progression of keratoconus.

\section{Subjects And Methods}

\section{Subjects}

Individuals with keratoconus who visited Ohshima Eye Hospital from April 2014 through July 2019 were candidates for this clinical study. Out of these 476 keratoconus patients, those who met the following criteria were actually eligible for enrollment in the study: (1) wearer of a conventional HCL such as MILD II or MILDUV (Suncon, Kyoto, Japan), (2) underwent AS-OCT examination, and (3) underwent preferred HCL fitting for a keratoconic cornea. We determined the conditions of preferred HCL fitting on the basis of the following factors: (1) ability to wear an $\mathrm{HCL}$ on a keratoconic cornea for $>8 \mathrm{~h}$ per day, (2) absence of a foreign body sensation that made lens wear difficult, and (3) the HCL covers the pupil area during wear. Individuals with ocular diseases other than keratoconus, with eyelid diseases, or with a history of ocular surgery were excluded. A total of 198 eyes of 131 keratoconus patients ( 135 eyes of 88 males and 63 eyes of 43 females; mean age \pm SD of $40.0 \pm 12.5$ years and age range of 16 to 70 years) was eventually

enrolled in the study. To develop the prediction formula, we selected the 102 eyes of 68 subjects (45 males and 23 females, with a mean age \pm SD of $41.2 \pm 13.2$ years and age range of 16 to 70 years) examined from April 2014 through March 2017 as the development group. We selected the 96 eyes of 63 subjects (43 males and 20 females, with a mean age \pm SD of $38.7 \pm 11.7$ years and age range of 18 to 70 years) examined from April 2017 through July 2019 as a validation group to validate the prediction model. This retrospective observational study was approved by the Institutional Review Board of Ohshima Eye Hospital (approval no. OEH-2019-03) and adhered to the tenets of the Declaration of Helsinki. Informed consent was obtained from all subjects.

\section{Collection of Clinical Information}

All subjects underwent three examinations by AS-OCT (CASIA; TOMEY, Nagoya, Japan) under the noncycloplegic condition. Twenty minutes after removal of HCLs, eyes were examined three times by examiners who were unaware of the study. A decimal visual acuity chart with Landolt rings was used to 
perform the measurements. Actual HCL-BCVA was evaluated by the following protocol: (1) measurement of ocular refraction during HCL wear, and (2) measurement of HCL-BCVA by the cross-cylinder method based on the measurement of (1). Clinical parameters measured by AS-OCT for development of the prediction model were corneal thickness $(\mu \mathrm{m})$, anterior corneal refractive power $(D)$, anterior corneal surface astigmatism (D), anterior corneal surface asymmetry (D), anterior corneal surface higher astigmatism (D), posterior corneal refractive power (D), posterior corneal surface astigmatism (D), posterior corneal surface asymmetry (D), posterior corneal surface higher astigmatism (D), spherical aberration (D), and high-order aberrations $(\mu \mathrm{m})$.

\section{Development of a Prediction Model}

On the basis of the collected clinical data, we developed a prediction model for HCL-BCVA by applying multiple regression analysis. We first designated the following 13 factors as independent variables: sex, age, corneal thickness, anterior corneal refractive power, anterior corneal surface astigmatism, anterior corneal surface asymmetry, anterior corneal surface higher astigmatism, posterior corneal refractive power, posterior corneal surface astigmatism, posterior corneal surface asymmetry, posterior corneal surface higher astigmatism, spherical aberration (SA), and high-order aberrations (HOAs). HCL-BCVA was considered a dependent (objective) variable. We then applied stepwise multiple regression analysis to identify independent variables that significantly affect prediction of the dependent variable.

\section{Statistical Analysis}

Decimal visual acuity was changed to logMAR visual acuity for statistical analysis. As described above, we applied stepwise multiple regression analysis to identify statistically significant independent variables for prediction of HCL-BCVA. Multicollinearity between the selected independent variables had a variance inflation factor of $<10$. The accuracy of each potential prediction formula was evaluated by Pearson's correlation coefficient. A $P$ value of $<0.05$ was considered statistically significant. All statistical analysis was performed with IBM SPSS Statistics software version 24 (IBM, Armonk, NY).

\section{Results}

\section{Development of a Prediction Model}

With the clinical corneal parameter values obtained from the development group, we developed a formula to predict HCL-BCVA. The statistical method of stepwise multiple regression analysis provides the best combination of suggested independent variables for indication of dependent variables. Such analysis identified anterior corneal refractive power, age, HOAs, anterior corneal surface asymmetry, and posterior corneal surface asymmetry as the best combination of such independent variables (Table 1). On the basis of the stepwise multiple regression analysis, we developed the following multiple regression equation for HCL-BCVA: 
Table 1

Stepwise multiple regression analysis for prediction of HCL-BCVA in the development group $(n=102$ eyes of 68 keratoconus patients)

\begin{tabular}{|llllll|}
\hline Parameter & $\begin{array}{l}\text { Regression } \\
\text { coefficient }\end{array}$ & $\begin{array}{l}\text { Standard } \\
\text { error }\end{array}$ & $\begin{array}{l}\boldsymbol{t} \\
\text { ratio }\end{array}$ & $\begin{array}{l}\boldsymbol{P} \text { value } \\
\text { variance }\end{array}$ & $\begin{array}{l}\text { Varion } \\
\text { inflation } \\
\text { factor }\end{array}$ \\
\hline Intercept & -0.674 & 0.140 & - & $<$ & \\
\hline $\begin{array}{l}\text { Anterior corneal refractive } \\
\text { power }\end{array}$ & 0.011 & 0.003 & 3.663 & $<$ & 2.167 \\
\hline Age & 0.002 & 0.001 & 2.409 & 0.018 & 1.139 \\
\hline $\begin{array}{l}\text { High-order aberrations } \\
\text { Anterior corneal surface }\end{array}$ & 0.079 & 0.020 & 3.886 & $<$ & 2.892 \\
\hline $\begin{array}{l}\text { Psymmetry } \\
\text { Posterior corneal surface }\end{array}$ & -0.030 & 0.008 & - & & \\
asymmetry & 0.054 & 0.026 & 2.101 & 0.038 & 1.986 \\
\hline
\end{tabular}

Predicted HCL-BCVA value $=-0.674+(0.011 \times$ anterior corneal refractive power [D] $)+(0.002 \times$ age $)+$ $(0.079 \times \mathrm{HOAs}[\mu \mathrm{m}])+(-0.030 \times$ anterior corneal surface asymmetry [D] $)+(0.054 \times$ posterior corneal surface asymmetry[D])

The distribution of actual and predicted HCL-BCVA values for the eyes in the development group is shown in Fig. 1. The correlation coefficient for the multiple regression equation was $0.728\left(R^{2}=0.530\right)$. The error average of the equation was -0.003 , and the root mean squared residual (RMSR) was 0.120 (Table 2). Statistical analysis revealed that the $P$ value for the equation was $<0.001$.

Table 2

Model performance for prediction of HCL-BCVA compared with actual HCL-BCVA in the development group ( $n=102$ eyes of 68 keratoconus patients)

\begin{tabular}{|lccccc|}
\hline & Mean & SD & Minimum & Median & Maximum \\
\hline Actual HCL-BCVA & 0.093 & 0.017 & -0.180 & 0.000 & 0.700 \\
\hline Predicted HCL-BCVA & 0.096 & 0.128 & -0.012 & 0.077 & 0.516 \\
\hline Residual error for HCL-BCVA & -0.003 & 0.120 & -0.368 & -0.010 & 0.476 \\
\hline Root mean squared residual $=0.120$. & & & & \\
\hline
\end{tabular}

\section{Validation of the Prediction Model}

To validate the prediction equation for HCL-BCVA, we applied the clinical information for the validation group to the model and then compared the predicted HCL-BCVA values with the actual HCL-BCVA values. 
The distribution of the predicted and actual HCL-BCVA values for the eyes in the validation group is shown in Fig. 2. The correlation coefficient for actual versus predicted values was $0.641\left(R^{2}=0.411\right)$. The error average of the correlation curve was 0.002 , and the RMSR was 0.099 (Table 3). Statistical analysis revealed that the $P$ value for the multiple regression equation for predicted and actual HCL-BCVA was < 0.001. The percentage of eyes with a difference between actual and predicted HCL-BCVA of within $\log M A R \pm 0.1$ or $\log M A R \pm 0.2$ was $71.9 \%$ (69 out of 96 eyes) and $96.9 \%$ (93 out of 96 eyes), respectively (Fig. 2).

Table 3

Validation of model performance for prediction of HCL-BCVA compared with actual HCL-BCVA in the validation group ( $n=96$ eyes of 63 keratoconus patients)

\begin{tabular}{|lccccc|}
\hline & Mean & SD & Minimum & Median & Maximum \\
\hline Actual HCL-BCVA & 0.065 & 0.011 & -0.180 & 0.025 & 0.400 \\
\hline Predicted HCL-BCVA & 0.063 & 0.123 & -0.090 & 0.024 & 0.521 \\
\hline Residual error for HCL-BCVA & 0.002 & 0.099 & -0.301 & 0.004 & 0.218 \\
\hline \multicolumn{2}{l}{ Root mean squared residual $=0.099}$. & & & & \\
\hline
\end{tabular}

\section{Single Regression Analysis and Cutoff Values}

We performed simple regression analysis to generate regression curves for evaluation of the relation between actual HCL-BCVA and clinical corneal parameters for all 198 eyes enrolled in the study. HCLBCVA was set as an objective variable, and 13 clinical parameters-corneal thickness, anterior corneal refractive power, anterior corneal surface astigmatism, anterior corneal surface asymmetry, anterior corneal surface higher astigmatism, posterior corneal refractive power, posterior corneal surface astigmatism, posterior corneal surface asymmetry, posterior corneal surface higher astigmatism, SA, HOAs, sex, and age-were selected as explanatory variables. Simple regression analysis revealed that all clinical parameters with the exception of sex showed a significant relation $(P<0.05)$ to HCL-BCVA (Table 4). From among these significant clinical parameters, we selected the three with an absolute $R$ value of $>$ 0.5 , those were sufficient to identify the linear relationship-anterior corneal refractive power $(R=0.606)$, posterior corneal refractive power $(R=-0.617)$, and HOAs $(R=0.506)$-for further evaluation of their relation to HCL-BCVA. The regression curves for HCL-BCVA and anterior corneal refractive power (Fig. 3), posterior corneal refractive power (Fig. 4), or HOAs (Fig. 5) were linear, with cutoff values for the three selected parameters corresponding to a logMAR of 0.15 for HCL-BCVA being 57.18D, - 8.16D, and 1.71 $\mu \mathrm{m}$, respectively. 
Table 4

Single regression analysis for the relation between actual HCL-BCVA and clinical factors for all study subjects ( $n=198$ eyes of 131 keratoconus patients)

\begin{tabular}{|lll|}
\hline Clinical factor & Correlation coefficient & $P$ value \\
\hline Age & 0.254 & $<0.001$ \\
\hline Sex & 0.092 & 0.198 \\
\hline Anterior corneal refractive power & -0.403 & $<0.001$ \\
\hline Anterior corneal surface astigmatism & 0.606 & $<0.001$ \\
\hline Posterior corneal refractive power & 0.240 & $<0.001$ \\
\hline Posterior corneal surface astigmatism & -0.617 & $<0.001$ \\
\hline Anterior corneal surface asymmetry & 0.226 & 0.001 \\
\hline Anterior corneal surface higher astigmatism & 0.153 & 0.032 \\
\hline Posterior corneal surface asymmetry & 0.369 & $<0.001$ \\
\hline Posterior corneal surface higher astigmatism & 0.388 & $<0.001$ \\
\hline Spherical aberration & -0.385 & $<0.001$ \\
\hline High-order aberrations & 0.506 & $<0.001$ \\
\hline
\end{tabular}

\section{Discussion}

In this study, we established a prediction formula for HCL-BCVA based on clinical parameters measured by AS-OCT, with the formula showing a reliability sufficient for prediction of HCL-BCVA for keratoconus patients. We also identified clinical parameters that are linearly related to HCL-BCVA and determined threshold values for these parameters associated with maintenance of a favorable HCL-BCVA. Our results thus suggest a new approach to determination of treatment strategy for progressive keratoconus.

The correlation coefficient between predicted HCL-BCVA and actual HCL-BCVA was 0.641 for the validation group. Furthermore, the prediction error ratio for HCL-BCVA within logMAR values of \pm 0.1 or \pm 0.2 was $71.9 \%$ and $96.9 \%$, respectively, which is sufficient for application to the clinical setting. With the use of our prediction formula, it is thus possible to predict HCL-BCVA without HCL wear. Similar studies have previously been performed to predict BCVA for keratoconus patients wearing glasses. ${ }^{23-26}$ For keratoconus patients, however, because of their corneal irregular astigmatism, HCL-BCVA is usually better than BCVA with glasses. ${ }^{3}$ Prediction of HCL-BCVA is thus more relevant to the clinical setting for individuals with keratoconus. 
We also performed simple regression analysis to identify parameters that are linearly related to HCLBCVA of keratoconus patients. Our analysis revealed that the relation between actual HCL-BCVA and anterior corneal refractive power, posterior corneal refractive power, or HOAs was linear. On the basis of the regression lines for these relations, we calculated cutoff (threshold) values for the three parameters corresponding to a logMAR value of 0.15 for HCL-BCVA, which is the value required for renewal of a driving license in Japan and is a good indicator for quality of life. The cutoff values for anterior corneal refractive power, posterior corneal refractive power, and HOAs were 57.18D, $-8.16 \mathrm{D}$, and $1.71 \mu \mathrm{m}$, respectively.

CXL is currently applied to prevent the progression of keratoconus, ${ }^{21}$ with many studies having supported its clinical efficacy. ${ }^{21,27-35}$ Several criteria for performance of CXL have been proposed, ${ }^{22}$ but they are all based on the degree of progression of keratoconus. The development of keratoconus tends to occur at a relatively young age, with affected individuals being able to maintain an active lifestyle if their vision remains good. Young patients are more tolerant of HCLs, and it is important that they be treated to prevent the progression of keratoconus while they are still able to achieve good vision while wearing such lenses. While we agree that confirmation of disease progression is a requirement for administration of CXL treatment, we have now shown that several clinical parameters manifest threshold values with regard to loss of HCL-BCVA. In particular, if keratoconus patients cross thresholds of 57.18D for anterior corneal refractive power, $-8.16 \mathrm{D}$ for posterior corneal refractive power, or $1.71 \mu \mathrm{m}$ for HOAs, then they may no longer be able to maintain an adequate HCD-BCVA and may therefore become candidates for corneal surgery such as keratoplasty. Although clinical results for keratoplasty in individuals with keratoconus are good, ${ }^{36-39}$ such surgery is associated with several complications and should be avoided if possible. ${ }^{40,41}$ We thus propose that the threshold values for clinical parameters identified in the present study on the basis of clinical data of many keratoconus patients should be considered in selecting treatment strategy for this condition. These thresholds should thus be taken into account in determining the surgical indication for $C X L$, so that the procedure can be performed before patients cross the line with regard to the potential for achieving a good HCL-BCVA.

Our study has at least a couple of limitations. First, AS-OCT was performed with the CASIA system, which is not able to provide separate values for anterior and posterior HOAs and for anterior and posterior SA, but instead provides only corresponding total values. In addition, the parameters considered for development of our prediction formula did not include coma aberration, a specific parameter of keratoconic corneas, as a result of limitations of the AS-OCT settings. Second, given the study setting, we evaluated HCL-BCVA only with conventional spherical HCLs, not with keratoconus-specific HCLs. The correction efficacy of keratoconus-specific HCLs usually differs from that of conventional spherical HCLs, with further studies with keratoconus-specific HCLs thus being warranted.

In conclusion, we have developed a formula to predict HCL-BCVA in keratoconus patients. We also identified three clinical parameters-anterior corneal refractive power, posterior corneal refractive power, and HOAs - that are linearly related to HCL-BCVA in such patients, and we determined cutoff values for these three parameters that are associated with the ability to achieve a favorable HCL-BCVA. Our 
approach to prediction of HCL-BCVA in keratoconus patients on the basis of AS-OCT measurements should prove helpful for management of this condition, and the cutoff values of the three identified clinical parameters should be taken into account when considering the indications for CXL and other treatments.

\section{Declarations}

\section{Ethics approval and consent to participate:}

Internal Review Board of Ohshima Eye Hospital approved this clinical study. Informed consent was obtained from all participates.

\section{Consent for publication:}

All authors agreed this manuscript to be published.

\section{Availability of data and materials:}

Row data was available at: https://figshare.com/articles/dataset/BMC_magome_xlsx/16528569

\section{Competing interests:}

none

\section{Funding:}

none

\section{Authors' contributions:}

$\mathrm{K}$. Magome and N. Morishige wrote the main manuscript text, K. Magome and $\mathrm{H}$. Nagai collected the data, A. Ueno and T. Matsui reviewed the manuscript and provided critical comments and and E. Uchio organized this study.

\section{Acknowledgements:}

none 


\section{References}

1. Bawazeer AM, Hodge WG,Lorimer B. Atopy and keratoconus: a multivariate analysis. $\mathrm{Br} \mathrm{J}$ Ophthalmol. 2000; 84: 834-836.

2. Bron AJ. Keratoconus. Cornea. 1988; 7: 163-169.

3. Krachmer JH, Feder RS,Belin MW. Keratoconus and related noninflammatory corneal thinning disorders. Surv Ophthalmol. 1984; 28: 293-322.

4. Lim N,Vogt U. Characteristics and functional outcomes of 130 patients with keratoconus attending a specialist contact lens clinic. Eye (Lond). 2002; 16: 54-59.

5. Maeda N, Fujikado T, Kuroda T, et al. Wavefront aberrations measured with Hartmann-Shack sensor in patients with keratoconus. Ophthalmology. 2002; 109: 1996-2003.

6. Rabinowitz YS. Keratoconus. Surv Ophthalmol. 1998; 42: 297-319.

7. Toprak I, Yaylali V,Yildirim C. A combination of topographic and pachymetric parameters in keratoconus diagnosis. Cont Lens Anterior Eye. 2015; 38: 357-362.

8. Barbero S, Marcos S,Merayo-Lloves J. Corneal and total optical aberrations in a unilateral aphakic patient. J Cataract Refract Surg. 2002; 28: 1594-1600.

9. Choi J, Wee WR, Lee JH, et al. Changes of ocular higher order aberration in on- and off-eye of rigid gas permeable contact lenses. Optom Vis Sci. 2007; 84: 42-51.

10. Dorronsoro C, Barbero S, Llorente L, et al. On-eye measurement of optical performance of rigid gas permeable contact lenses based on ocular and corneal aberrometry. Optom Vis Sci. 2003; 80: 115125.

11. Downie LE,Lindsay RG. Contact lens management of keratoconus. Clin Exp Optom. 2015; 98: 299311.

12. Hong $X$, Himebaugh $N$,Thibos LN. On-eye evaluation of optical performance of rigid and soft contact lenses. Optom Vis Sci. 2001; 78: 872-880.

13. Kosaki R, Maeda N, Bessho K, et al. Magnitude and orientation of Zernike terms in patients with keratoconus. Invest Ophthalmol Vis Sci. 2007; 48: 3062-3068.

14. Mannis MJ,Zadnik K. Contact lens fitting in keratoconus. CLAO J. 1989; 15: 282-289.

15. Negishi K, Kumanomido T, Utsumi Y, et al. Effect of higher-order aberrations on visual function in keratoconic eyes with a rigid gas permeable contact lens. Am J Ophthalmol. 2007; 144: 924-929.

16. Betts AM, Mitchell GL,Zadnik K. Visual performance and comfort with the Rose K lens for keratoconus. Optom Vis Sci. 2002; 79: 493-501.

17. Jain AK,Sukhija J. Rose-K contact lens for keratoconus. Indian J Ophthalmol. 2007; 55: 121-125.

18. Schornack MM,Patel SV. Scleral lenses in the management of keratoconus. Eye Contact Lens. 2010; 36: 39-44.

19. Chen M,Yoon G. Posterior corneal aberrations and their compensation effects on anterior corneal aberrations in keratoconic eyes. Invest Ophthalmol Vis Sci. 2008; 49: 5645-5652. 
20. Nakagawa T, Maeda N, Kosaki R, et al. Higher-order aberrations due to the posterior corneal surface in patients with keratoconus. Invest Ophthalmol Vis Sci. 2009; 50: 2660-2665.

21. Wollensak G, Spoerl E,Seiler T. Riboflavin/ultraviolet-a-induced collagen crosslinking for the treatment of keratoconus. Am J Ophthalmol. 2003; 135: 620-627.

22. Hersh PS, Stulting RD, Muller D, et al. United States Multicenter Clinical Trial of Corneal Collagen Crosslinking for Keratoconus Treatment. Ophthalmology. 2017; 124: 1259-1270.

23. Esaka Y, Kojima T, Dogru M, et al. Prediction of Best-Corrected Visual Acuity With Swept-Source Optical Coherence Tomography Parameters in Keratoconus. Cornea. 2019; 38: 1154-1160.

24. Holladay JT. Corneal topography using the Holladay Diagnostic Summary. J Cataract Refract Surg. 1997; 23: 209-221.

25. Ucakhan 00. Predicted corneal visual acuity in keratoconus as determined by ray tracing. Acta Ophthalmol Scand. 2003; 81: 264-270.

26. Wilson SE,Klyce SD. Quantitative descriptors of corneal topography. A clinical study. Arch Ophthalmol. 1991; 109: 349-353.

27. Aixinjueluo W, Usui T, Miyai T, et al. Accelerated transepithelial corneal cross-linking for progressive keratoconus: a prospective study of 12 months. Br J Ophthalmol. 2017; 101: 1244-1249.

28. Beshtawi IM, Akhtar R, Hillarby MC, et al. Biomechanical properties of human corneas following lowand high-intensity collagen cross-linking determined with scanning acoustic microscopy. Invest Ophthalmol Vis Sci. 2013; 54: 5273-5280.

29. Gokul A, Vellara HR,Patel DV. Advanced anterior segment imaging in keratoconus: a review. Clin Exp Ophthalmol. 2018; 46: 122-132.

30. Kato N, Konomi K, Shinzawa M, et al. Corneal crosslinking for keratoconus in Japanese populations: one year outcomes and a comparison between conventional and accelerated procedures. Jpn J Ophthalmol. 2018; 62: 560-567.

31. Kato N, Negishi K, Sakai C, et al. Five-year Outcomes of Corneal Cross-Linking for Keratoconus: Comparison Between Conventional and Accelerated Procedures. Cornea. 2020; 39: e1.

32. Martin R. Cornea and anterior eye assessment with placido-disc keratoscopy, slit scanning evaluation topography and scheimpflug imaging tomography. Indian J Ophthalmol. 2018; 66: 360366.

33. Mita M, Waring GOt,Tomita M. High-irradiance accelerated collagen crosslinking for the treatment of keratoconus: six-month results. J Cataract Refract Surg. 2014; 40: 1032-1040.

34. Shinzawa M, Kato N, Kasai K, et al. Corneal cross-linking for keratoconus caused by compulsive eye rubbing in patients with Tourette syndrome: Three case reports. Medicine (Baltimore). 2019; 98: e15658.

35. Tomita M, Mita M,Huseynova T. Accelerated versus conventional corneal collagen crosslinking. J Cataract Refract Surg. 2014; 40: 1013-1020. 
36. Gulias-Canizo R, Gonzalez-Salinas R, Hernandez-Zimbron LF, et al. Indications and outcomes of pediatric keratoplasty in a tertiary eye care center: A retrospective review. Medicine (Baltimore). 2017; 96: e8587.

37. Ono T, Ishiyama S, Hayashidera T, et al. Twelve-year follow-up of penetrating keratoplasty. Jpn J Ophthalmol. 2017; 61: 131-136.

38. Parker JS, van Dijk K,Melles GR. Treatment options for advanced keratoconus: A review. Surv Ophthalmol. 2015; 60: 459-480.

39. Tan DT, Janardhanan P, Zhou H, et al. Penetrating keratoplasty in Asian eyes: the Singapore Corneal Transplant Study. Ophthalmology. 2008; 115: 975-982 e971.

40. Ibrahim O, Yagi-Yaguchi Y, Kakisu K, et al. Association of Iris Damage With Reduction in Corneal Endothelial Cell Density After Penetrating Keratoplasty. Cornea. 2019; 38: 268-274.

41. Sarnicola V, Toro P, Gentile D, et al. Descemetic DALK and predescemetic DALK: outcomes in 236 cases of keratoconus. Cornea. 2010; 29: 53-59.

\section{Figures}




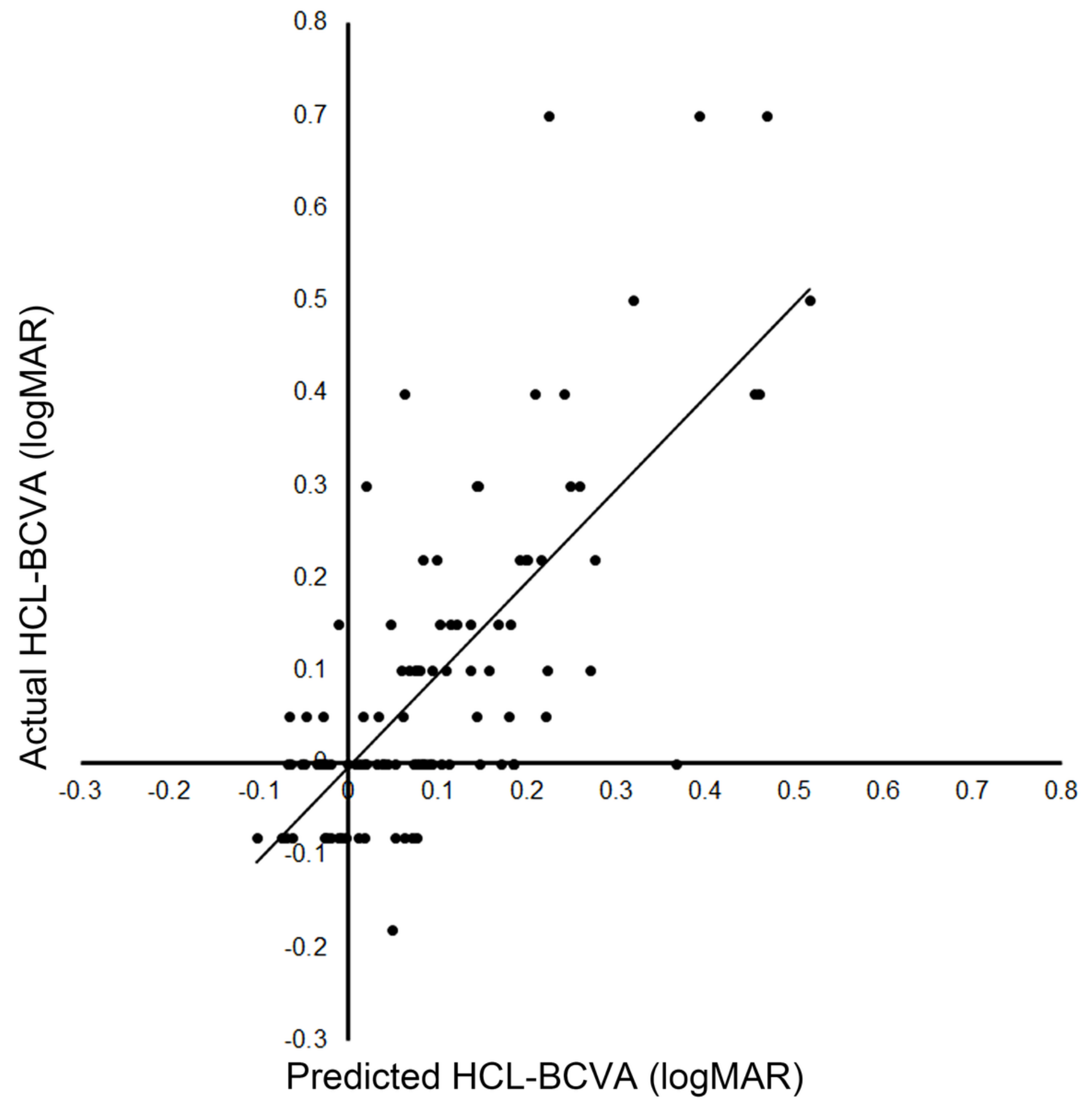

Figure 1

Distribution of actual HCL-BCVA and predicted HCL-BCVA in the development group ( $n=102$ eyes of 68 keratoconus patients). Predicted HCL-BCVA was correlated with actual HCL-BCVA (R2 = 0.530). 


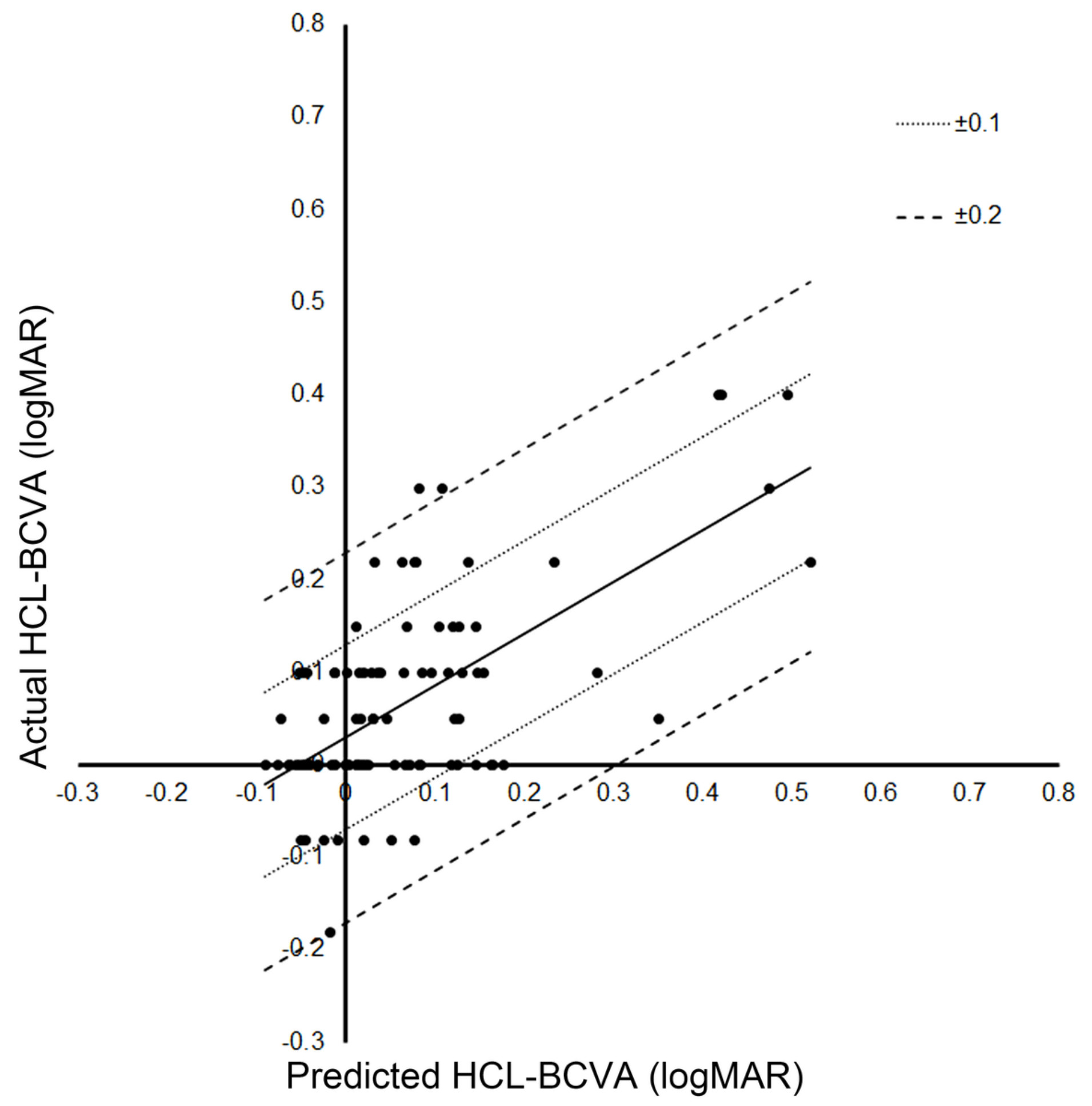

Figure 2

Distribution of actual HCL-BCVA and predicted HCL-BCVA in the validation group ( $\mathrm{n}=96$ eyes of 63 keratoconus patients). Predicted HCL-BCVA was correlated with actual HCL-BCVA (R2 = 0.411). Dotted and dashed lines indicate the regression lines for $\log M A R \otimes 0.1$ and $\otimes 0.2$, respectively. 


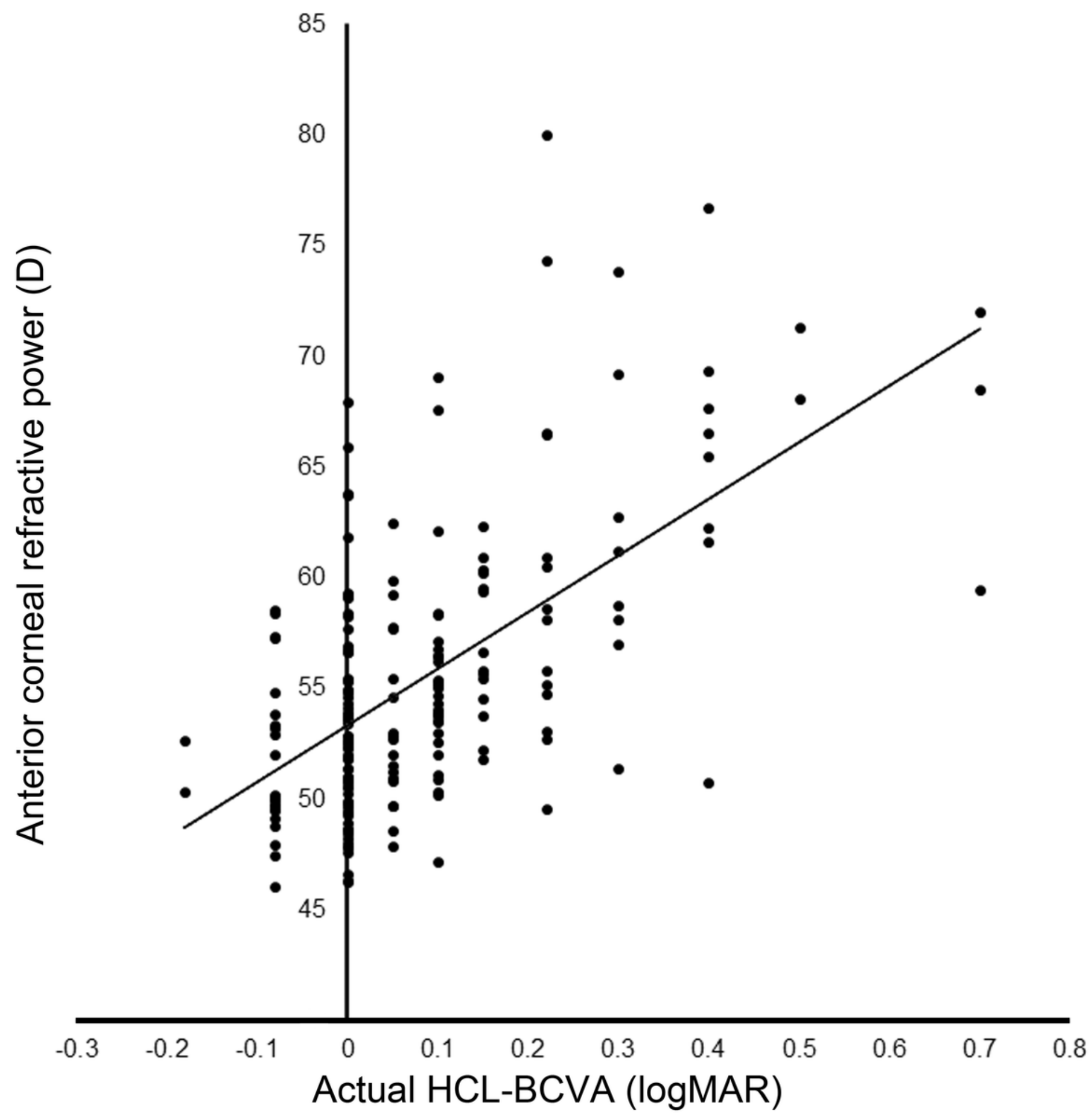

Figure 3

Relation between actual HCL-BCVA and anterior corneal refractive power for all study subjects $(n=198$ eyes of 131 keratoconus patients). The equation for the regression line is $y=25.606 x+53.334$ (R2 $=$ $0.367)$. 


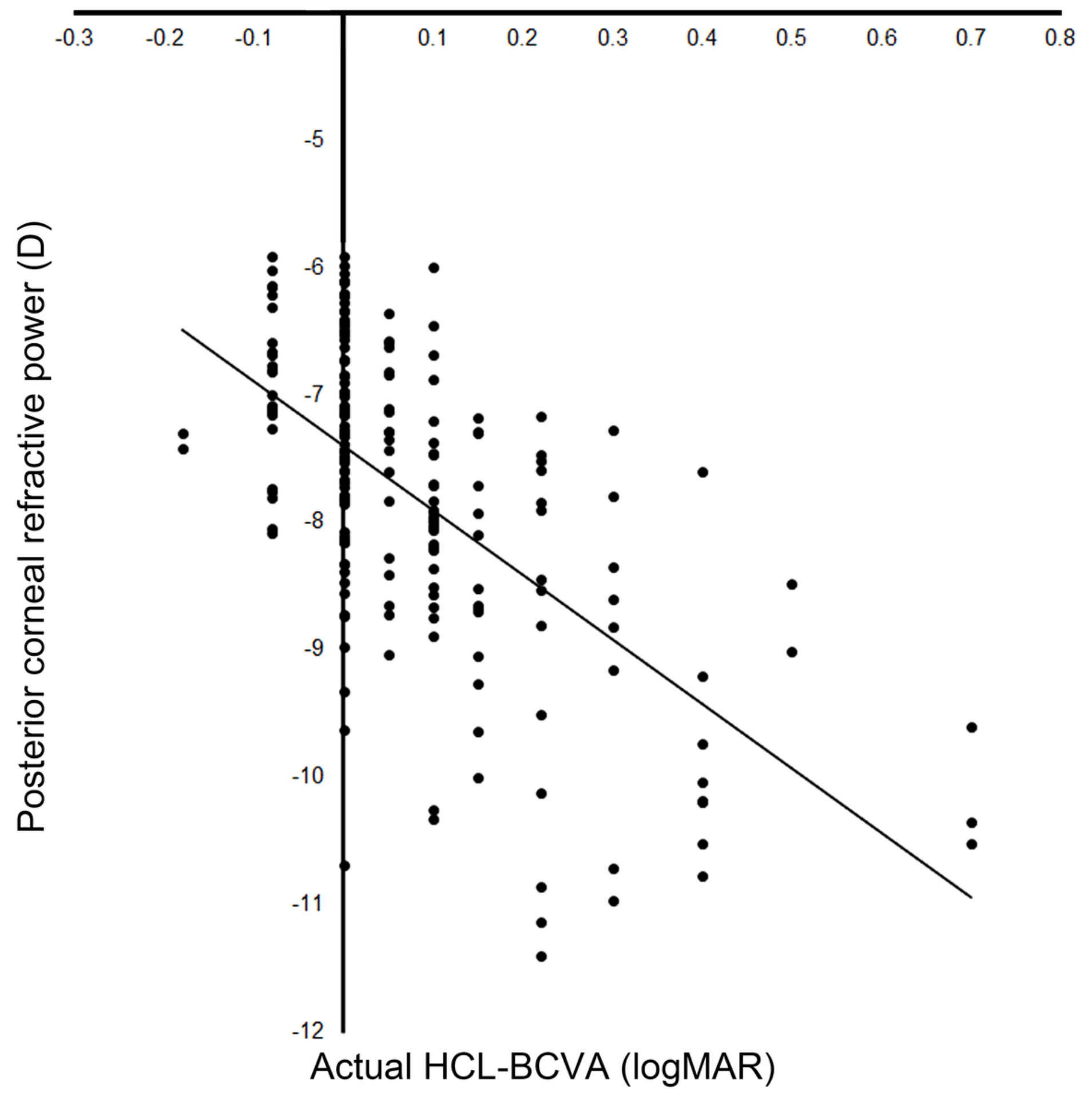

Figure 4

Relation between actual HCL-BCVA and posterior corneal refractive power for all study subjects $(n=198$ eyes of 131 keratoconus patients). The equation for the regression line is $y=-5.0418 x-7.404$ (R2 = $0.381)$. 


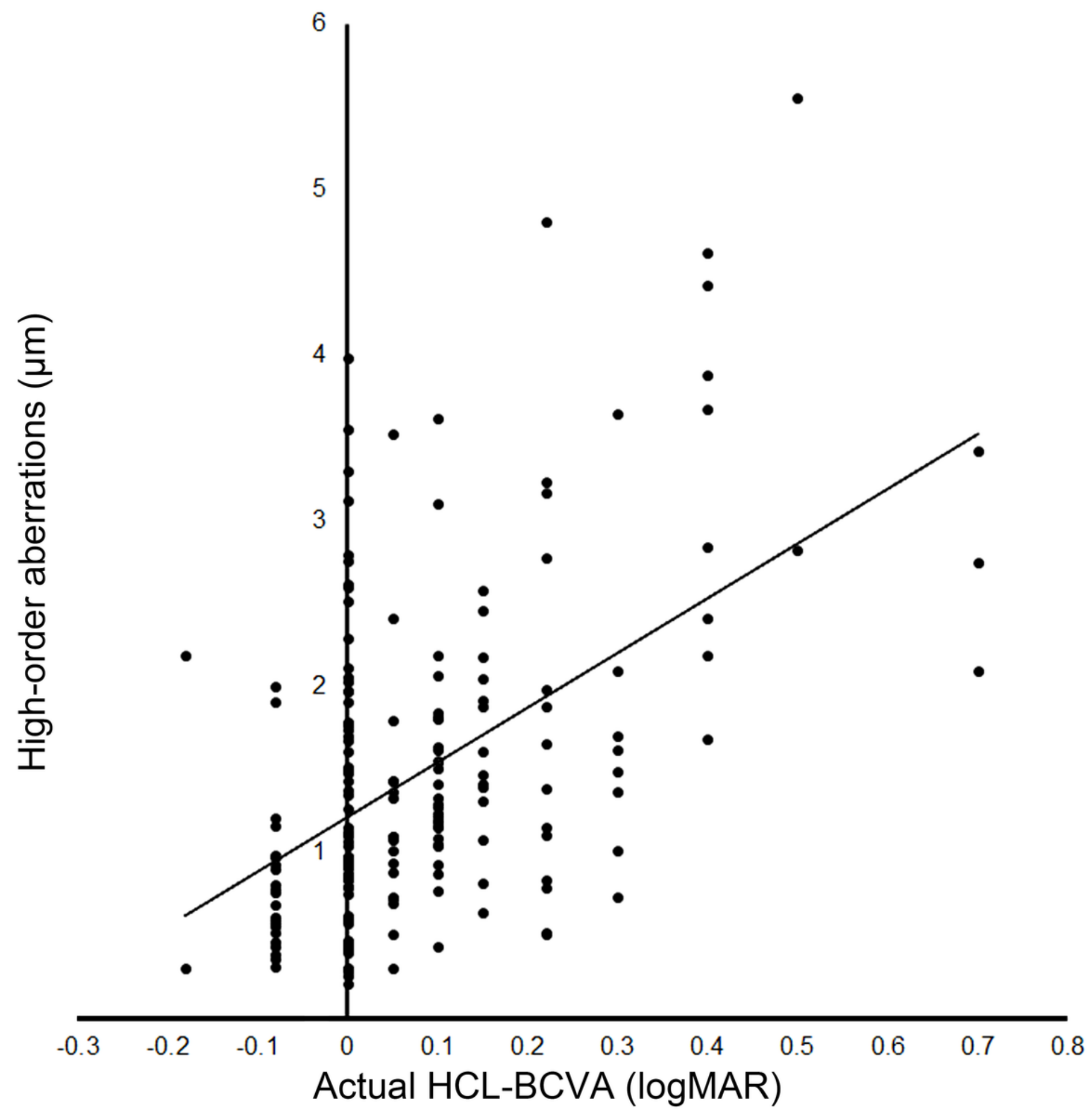

Figure 5

Relation between actual HCL-BCVA and high-order aberrations for all study subjects ( $\mathrm{n}=198$ eyes of 131 keratoconus patients). The equation for the regression line is $y=3.303 x+1.21133(R 2=0.256)$. 